\title{
Quantum Lorentz Group Having Gauss Decomposition Property
}

\author{
By
}

\author{
S. L. WORONOWICZ* ${ }^{* \dagger}$ and S. ZAKRZEWSKI*‡
}

\begin{abstract}
A new deformation of $S L(2, \mathrm{C})$ (considered as a real Lie group) is constructed and shown to have a Gauss type decomposition. The groups entering this decomposition are identified as $E_{\mu}(2)$ and its Pontryagin dual $\hat{E}_{\mu}(2)$. The whole group is the double group built over $E_{\mu}(2)$.
\end{abstract}

\section{§0. Introduction}

The first quantum deformation of Lorentz group was described in [5]. The theory presented in that paper based on the following four assumptions:

1. The tensor square of the (two-dimensional) spinor representation splits into a direct sum of two components, one of which is a one-dimensional trivial representation.

2. The tensor product of the spinor representation by the complex conjugate one is irreducible and does not depend on the order of factors.

3. The quantum Lorentz group contains $S_{\mu} U(2)$ as a subgroup (Throughout the paper, $\mu \in] 0,1[$ denotes the deformation parameter).

4. The quantum Lorentz group is not a proper subgroup of a quantum group satisfying the above three conditions.

The role of the last assumption (which was used but not explicitely formulated in [5]) is explained in [9]. It was shown that the above requirements determine the quantum Lorentz group completely (in fact there are two solu-

Communicated by H. Araki, August 12, 1991.

1991 Mathematics Subject Classification: 20N99, 57T05.

* Permanent address: Department of Mathematical Methods in Physics, Faculty of Physics, University of Warsaw, Hoża 74, 00-682 Warszawa, Polska.

† ETH Zürich. Supported in equal parts by the grant of the Ministry of Education of Poland and by Schweizerischer Nationalfonds.

$\ddagger$ Supported in equal parts by the grant of the Ministry of Education of Poland and by Alexander von Humboldt Stiftung. 
tions, the choice consists in declaring which of the two spinor representation is the fundamental one).

It turns out that deleting the third assumption we still have a limited number of possibilities. We refer to our paper [9] where we classify (on the Hopf *-algebra level) all quantum groups of $2 \times 2$ matrices satisfying the assumptions 1,2 and 4. In the present paper we investigate in details one of the possibilities distinguished by the fact that the corresponding quantum Lorentz group exhibits Gauss-type decomposition.

The classical Gauss decomposition [2] says that except a closed set of measure zero any element $g$ of $S L(2, \mathbb{C})$ has a unique decomposition of the form

$$
g=n_{+} h n_{-}
$$

where $n_{+}\left(n_{-}\right.$resp.) belongs to the subgroup $N_{+}\left(N_{-}\right.$resp.) of all upper (lower resp.) triangular matrices with one on the diagonal and $h$ belongs to the subgroup $H$ of all diagonal matrices. In short.

$$
G^{\prime}=N_{+} H N_{-}
$$

where $G^{\prime}$ is an open full measure subset of $S L(2, \mathbb{C})$.

In the quantum case however the subgroups analogous to $N_{ \pm}$do not exist (because of the nontrivial commutation relations between diagonal and off-diagonal elements). To deal with this difficulty one may include the diagonal part of the decomposition into the both factors $N_{ \pm}$considering the decomposition of the form

$$
G^{\prime}=M_{+} M_{-}
$$

where $M_{+}\left(M_{-}\right.$resp.) is the subgroup of all upper (lower) triangular matrices. This form of Gauss decomposition was proposed by Faddeev, Reshetikhin and Takhtajan [4]. One should notice however that in this case the decomposition is not unique.

In this paper we shall prove the quantum version of the decomposition of the form

$$
G^{\prime}=G_{1} G_{2}
$$

where $G_{1}\left(G_{2}\right.$ resp.) is the subgroup of upper (lower resp.) triangular matrices having unitary (positive resp.) elements on the diagonal. Comparing with (1) we see that $H$ has been split into $H_{\text {unitary }}$ included into $N_{+}$and $H_{\text {positive }}$ included into $N_{-}$. We would like to point out that in certain sense the Gauss decomposition in the quantum case is more satisfactory than the classical one: due to the commutation relations the diagonal elements are invertible so the decomposition has the global character with no exceptions. 
$G_{1}$ is the (double covering) of the group of motions of the euclidean plane, $G_{2}$ is isomorphic to the group of transformations of the plane generated by dilations and translations. In the quantum case the second group is the Pontriagin dual of the first one. Due to this fact we may use the double group construction (cf. [3], [5]). As a result we get the quantum Lorentz group on the $C^{*}$-level. In what follows this group will be called $G_{\mu}$.

The paper is composed in the following way. In Section 1 we introduce the Hopf *-algebra being a quantum deformation of the polynomial algebra on $S L(2, \mathrm{C})$. It is generated by four elements $\alpha, \beta, \gamma$ and $\delta$ satisfying certain commutation relations. The relations completed by a spectral condition formulated at the beginning of Section 4 are the main input of our theory. The anatomy of the commutation relations is investigated in Section 2. The main aim of this Section is to assign the strong meaning to the relations: since the involved elements are unbounded, one has to define carefully the domains where the relations are supposed to hold. In Section 3 we consider the Hilbert space representations of our relations. The main result shows that $\alpha$ and $\delta$ are invertible and that $\operatorname{Sp} \beta \gamma$ exhibits a radius quantization. Section 4 is devoted to the Gauss decomposition. In Section 5 we repeat the double group construction of [5] replacing the Iwasawa decomposition by that of Gauss to achieve our main goal. Namely we define the universal $C^{*}$-algebra $A$ "generated" by four elements $\alpha, \beta, \gamma$ and $\delta$ affiliated with it, satisfying the commutation relations and the spectral condition. It plays the role of the algebra of "all continuous functions on $G_{\mu}$ vanishing at infinity". The algebra $A$ is equipped with the comultiplication $\Phi \in \operatorname{Mor}(A, A \otimes A)$ acting on the generators in the standard way.

The notation used in this paper is explained in [7] and [8]. In particular

$$
\overline{\mathbf{C}}^{\mu}=\left\{t \in \mathbb{C}: t=0 \text { or }|t| \in \mu^{\mathbb{Z}}\right\},
$$

$F_{\mu}(\cdot)$ is the continuous function on $\overline{\mathbf{C}}^{\mu}$ introduced by

$$
F_{\mu}(t)=\prod_{k=0}^{\infty} \frac{1+\mu^{2 k} \bar{t}}{1+\mu^{2 k} t} .
$$

All $C^{*}$-algebras are concrete (i.e: acting on Hilbert spaces). For any Hilbert space $H, C^{*}(H)$ denotes the set of all $C^{*}$-algebras contained in $B(H)$. Let $A \in C^{*}\left(H_{A}\right), B \in C^{*}\left(H_{B}\right)$ and $\phi$ be a representation of $A$ acting on $H_{B}$. We remind that $\phi \in \operatorname{Mor}(A, B)$ if and only if $\phi(A) B$ is contained and dense in $B$. The symbol " $\eta$ " denotes the $C^{*}$-affiliation relation ([1], [6]): for any $A \in$ $C^{*}(H)$ and any closed operator $T$ acting on $H, T \eta A$ if and only if

$$
\begin{aligned}
& {\left[T\left(I+T^{*} T\right)^{-1 / 2}\right] A \subset A,} \\
& A\left[T\left(I+T^{*} T\right)^{-1 / 2}\right] \subset A,
\end{aligned}
$$




$$
\left[\left(I+T^{*} T\right)^{-1 / 2}\right] A \text { is dense in } A \text {. }
$$

The actions of morphisms admit the natural extension to the affiliated elements: if $\phi \in \operatorname{Mor}(A, B)$ and $T \eta A$ then $\phi(T)$ is a well defined element affiliated with $B$.

The closure of the sum of two operators $R$ and $S$ will be denoted by $R+S$.

\section{§1. Hopf *-algebra Level}

We fix deformation parameter $\mu \in] 0,1]$. Let $\mathscr{A}$ be the unital *-algebra generated by four elements $\alpha, \beta, \gamma$ and $\delta$ satisfying the following 17 relations:

$$
\begin{gathered}
\left.\alpha \beta=\mu \beta \alpha, \quad \begin{array}{c}
\beta \gamma=\gamma \beta, \\
\beta \gamma=\mu \delta \beta, \quad \gamma \delta=\mu \delta \gamma, \\
\alpha \delta-\mu \beta \gamma=I, \quad \delta \alpha-\mu^{-1} \beta \gamma=I
\end{array}\right\} \\
\alpha \beta^{*}=\mu \beta^{*} \alpha, \quad \beta \gamma^{*}=\gamma^{*} \beta, \quad \alpha \gamma^{*}=\mu^{-1} \gamma^{*} \alpha, \\
\beta \delta^{*}=\mu^{-1} \delta^{*} \beta, \quad \alpha \delta^{*}=\delta^{*} \alpha, \quad \gamma \delta^{*}=\mu \delta^{*} \gamma, \\
\alpha \alpha^{*}=\alpha^{*} \alpha, \quad \beta \beta^{*}=\beta^{*} \beta, \quad \gamma \gamma^{*}=\gamma^{*} \gamma, \\
\delta \delta^{*}=\delta^{*} \delta .
\end{gathered}
$$

The relations may be rewritten in a compact matrix form. Let $u=$ $\left(\begin{array}{ll}\alpha, & \beta \\ \gamma, & \delta\end{array}\right)$ and $\bar{u}=\left(\begin{array}{ll}\alpha^{*}, & \beta^{*} \\ \gamma^{*}, & \delta^{*}\end{array}\right)$. Then

$$
u \oplus u=\left(\begin{array}{llll}
\alpha \alpha, & \alpha \beta, & \beta \alpha, & \beta \beta \\
\alpha \gamma, & \alpha \delta, & \beta \gamma, & \beta \delta \\
\gamma \alpha, & \gamma \beta, & \delta \alpha, & \delta \beta \\
\gamma \gamma, & \gamma \delta, & \delta \gamma, & \delta \delta
\end{array}\right)
$$

and one can easily verify that relations (2) are equivalent to

$$
(u(1) u) E=E I \quad \text { and } \quad I E^{*}=E^{*}(u(1) u)
$$

where

$$
E=\left[\begin{array}{c}
0 \\
1 \\
-\mu \\
0
\end{array}\right] \quad \text { and } \quad E^{*}=(0,1,-\mu, 0)
$$

Similarly

$$
u \text { (1) } \bar{u}=\left(\begin{array}{llll}
\alpha \alpha^{*}, & \alpha \beta^{*}, & \beta \alpha^{*}, & \beta \beta^{*} \\
\alpha \gamma^{*}, & \alpha \delta^{*}, & \beta \gamma^{*}, & \beta \delta^{*} \\
\gamma \alpha^{*}, & \gamma \beta^{*}, & \delta \alpha^{*}, & \delta \beta^{*} \\
\gamma \gamma^{*}, & \gamma \delta^{*}, & \delta \gamma^{*}, & \delta \delta^{*}
\end{array}\right), \quad \bar{u}(1)=\left(\begin{array}{llll}
\alpha^{*} \alpha, & \alpha^{*} \beta, & \beta^{*} \alpha, & \beta^{*} \beta \\
\alpha^{*} \gamma, & \alpha^{*} \delta, & \beta^{*} \gamma, & \beta^{*} \delta \\
\gamma^{*} \alpha, & \gamma^{*} \beta, & \delta^{*} \alpha, & \delta^{*} \beta \\
\gamma^{*} \gamma, & \gamma^{*} \delta, & \delta^{*} \gamma, & \delta^{*} \delta
\end{array}\right) \text {. }
$$


and relations (3) are equivalent to

$$
X(\bar{u} \oplus u)=(u \oplus \bar{u}) X
$$

where

$$
X=\left(\begin{array}{llll}
\mu, & 0, & 0, & 0 \\
0, & 0, & 1, & 0 \\
0, & 1, & 0, & 0 \\
0, & 0, & 0 & \mu
\end{array}\right)
$$

Using the matrix form of the commutation relations one may prove

Theorem 1.1. 1. There exists unique *-algebra homomorphism

$$
\Phi: \mathscr{A} \rightarrow \mathscr{A} \otimes \mathscr{A}
$$

such that

$$
\left(\begin{array}{ll}
\Phi(\alpha), & \Phi(\beta) \\
\Phi(\gamma), & \Phi(\delta)
\end{array}\right)=u \oplus u=\left(\begin{array}{ll}
\alpha \otimes \alpha+\beta \otimes \gamma, & \alpha \otimes \beta+\beta \otimes \delta \\
\gamma \otimes \alpha+\delta \otimes \gamma, & \gamma \otimes \beta+\delta \otimes \delta
\end{array}\right) .
$$

2. There exists unique *-character

$$
e: \mathscr{A} \rightarrow \mathbf{C}
$$

such that

$$
\left(\begin{array}{ll}
e(\alpha), & e(\beta) \\
e(\gamma), & e(\delta)
\end{array}\right)=\left(\begin{array}{ll}
1, & 0 \\
0, & 1
\end{array}\right)
$$

3. There exists unique linear antimultiplicative mapping

$$
\kappa: \mathscr{A} \rightarrow \mathscr{A}
$$

such that

$$
\begin{gathered}
\left(\begin{array}{ll}
\kappa(\alpha), & \kappa(\beta) \\
\kappa(\gamma), & \kappa(\delta)
\end{array}\right)=u^{-1}=\left(\begin{array}{cc}
\delta, & -\mu^{-1} \beta \\
-\mu \gamma, & \alpha
\end{array}\right) \\
\left(\begin{array}{ll}
\kappa\left(\alpha^{*}\right), & \kappa\left(\beta^{*}\right) \\
\kappa\left(\gamma^{*}\right), & \kappa\left(\delta^{*}\right)
\end{array}\right)=(\bar{u})^{-1}=\left(\begin{array}{cc}
\delta^{*}, & -\mu \beta^{*} \\
-\mu^{-1} \gamma^{*}, & \alpha^{*}
\end{array}\right) .
\end{gathered}
$$

4. $(\mathscr{A}, \Phi, e, \kappa)$ is a Hopf*-algebra.

In the rest of the paper we shall construct a $C^{*}$-algebra "generated" by four distinguished elements $\alpha, \beta, \gamma$ and $\delta$ affiliated with it such that the relations (2) and (3) are satisfied in a certain strong sense. We shall also prove that there exists unique $C^{*}$-algebra category morphism $\Phi \in \operatorname{Mor}(A, A \otimes A)$ acting on distinguished elements $\alpha, \beta, \gamma$ and $\delta$ in the way described by (4). 


\section{§2. ( $p, q)$-commuting Normal Operators}

Throughout this section, $p$ and $q$ will denote two fixed strictly positive real numbers. The purpose of this section is to give a meaning to the following relations

$$
\begin{gathered}
R S=p S R, \\
R S^{*}=q S^{*} R,
\end{gathered}
$$

where $R$ and $S$ are normal (unbounded) operators acting on a Hilbert space $H$. We shall use the following.

Definition 2.1. Let $R$ and $S$ be normal operators acting on a Hilbert space $H$. We say that $(R, S)$ is a $(p, q)$-commuting pair if

1. $|R|$ and $|S|$ strongly commute

2. $\quad($ Phase $R)($ Phase $S)=($ Phase $S)($ Phase $R)$

3. On $(\operatorname{ker} R)^{\perp}$ we have

$$
(\text { Phase } R)|S|(\text { Phase } R)^{*}=\sqrt{p q}|S|
$$

4. On (key $S)^{\perp}$ we have

$$
(\text { Phase } S)|R|(\text { Phase } S)^{*}=\sqrt{q / p}|R| .
$$

The set of all $(p, q)$-commuting pairs of normal operators acting on a Hilbert space $H$ will be denoted by $\mathscr{C}_{H}(p, q)$ or simply by $\mathscr{C}(p, q)$ when it makes no confusion.

If $(R, S) \in \mathscr{C}(p, q)$ then the composition $R \circ S$ is closeable and its closure will be denoted by $R S$. One can easily verify that

$$
\begin{aligned}
\operatorname{Phase}(R S) & =(\text { Phase } R)(\text { Phase } S) \\
|R S| & =\sqrt{p / q}|R||S| .
\end{aligned}
$$

The operator $R S$ is normal if and only if $q=1$. Similarly one may consider products $S R, R^{*} S, S^{*} R$, etc.

Let $(R, S) \in \mathscr{C}(p, q)$. Then one can easily verify that

$$
\begin{gathered}
\left(R, S^{*}\right) \in \mathscr{C}(q, p) \\
(R, \text { Phase } S) \in \mathscr{C}(\sqrt{p / q}, \sqrt{q / p}) \\
(R,|S|) \in \mathscr{C}(\sqrt{p q}, \sqrt{p q}) \\
(S, R) \in \mathscr{C}\left(p^{-1}, q\right) .
\end{gathered}
$$

Moreover if $\operatorname{ker} S=\{0\}$ then

$$
\left(R, S^{-1}\right) \in \mathscr{C}\left(p^{-1}, q^{-1}\right)
$$


If $\left(R, S_{1}\right) \in \mathscr{C}\left(p_{1}, q_{1}\right),\left(R, S_{2}\right) \in \mathscr{C}\left(p_{2}, q_{2}\right)$ and $\left(S_{1}, S_{2}\right) \in \mathscr{C}\left(p_{3}, q_{3}\right)$ then using (6) to compute Phase $\left(S_{1} S_{2}\right)$ and $\left|S_{1} S_{2}\right|$ we see that

$$
\left(R, S_{1} S_{2}\right) \in \mathscr{C}\left(p_{1} p_{2}, q_{1} q_{2}\right)
$$

Similarly

$$
\left(R_{1} R_{2}, S\right) \in \mathscr{C}\left(p_{1} p_{2}, q_{1} q_{2}\right)
$$

provided $\left(R_{1}, S\right) \in \mathscr{C}\left(p_{1}, q_{1}\right),\left(R_{2}, S\right) \in \mathscr{C}\left(p_{2}, q_{2}\right)$ and $\left(R_{1}, R_{2}\right) \in \mathscr{C}\left(p_{3}, q_{3}\right)$.

Two normal operators $R$ and $S$ strongly commute (have joint spectral decomposition) if and only if $(R, S) \in \mathscr{C}(1,1)$.

We shall use the following version of Theorems 2.1 and 2.2 of [7].

Theorem 2.2. Let $(R, S) \in \mathscr{C}\left(\mu^{-2}, 1\right)$. Assume that $\operatorname{ker} R=\{0\}$. Then the following three conditions are equivalent:

(i) $R+S$ admits a normal extension,

(ii) $R+S$ is normal,

(iii) $\operatorname{Sp}\left(R^{-1} S\right) \subset \overline{\mathbf{C}}^{\mu}$.

Moreover, if one of the above conditions holds then $R+S$ is unitarily equivalent to $R$ :

$$
R+S=F_{\mu}\left(R^{-1} S\right) R F_{\mu}\left(R^{-1} S\right)^{*} .
$$

Proof. If $S=0$ then $R^{-1} S=0, F_{\mu}\left(R^{-1} S\right)=I$. Hence the conditions (i)(iii) are satisfied and the formula (14) holds. Therefore we may assume that ker $S=\{0\}$. In this case our theorem coincides with Theorem 2.1 of [7].

Q.E.D.

We also have

Theorem 2.3. Let $(R, S) \in \mathscr{C}\left(\mu^{-2}, 1\right)$. Assume that $\operatorname{ker} S=\{0\}$. Then the following three conditions are equivalent:

(i) $R+S$ admits a normal extension,

(ii) $R+S$ is normal,

(iii) $\operatorname{Sp}\left(R S^{-1}\right) \subset \overline{\mathbf{C}}^{\mu}$.

Moreover, if one of the above conditions holds then $R^{+1} S$ is unitarily equivalent to $S$ :

$$
R+S=F_{\mu}\left(R S^{-1}\right)^{*} S F_{\mu}\left(R S^{-1}\right) .
$$

Proof. If $R=0$ then $R S^{-1}=0, F_{\mu}\left(R S^{-1}\right)=I$. Hence the conditions (i)(iii) are satisfied and the formula (15) holds. Therefore we may assume that ker $R=\{0\}$. Then, by virtue of Theorem 2.2: (i) $\Leftrightarrow(\mathrm{ii}) \Leftrightarrow\left(\left(\operatorname{Sp}\left(R^{-1} S\right)\right) \subset \overline{\mathbf{C}}^{\mu}\right)$. Clearly the latter condition is equivalent to (iii) $\left(R S^{-1}=\mu^{2}\left(R^{-1} S\right)^{-1}\right.$, the transformation $t \mapsto \mu^{2} t^{-1}$ maps $\overline{\mathbf{C}}^{\mu}-\{0\}$ onto itself). 
Assume that $\operatorname{Sp}\left(R S^{-1}\right) \subset \overline{\mathbb{C}}^{\mu}$. Then $\operatorname{Sp}\left(\left(R S^{-1}\right)^{*}\right) \subset \overline{\mathbb{C}}^{\mu}$ and inserting in Theorem $2.2 S^{*}$ and $R^{*}$ instead of $R$ and $S$ we get

$$
R^{*} \dot{+} S^{*}=F_{\mu}\left(R S^{-1}\right)^{*} S^{*} F_{\mu}\left(R S^{-1}\right) .
$$

Clearly $(R+S)^{*} \supset\left(R^{*} \dot{+} S^{*}\right)$. Remembering that normal operators have no proper normal extensions we see that $(R \dot{+})^{*}=\left(R^{*} \dot{+} S^{*}\right)$. Now (15) follows immediately from (16).

Q.E.D.

Let $Q$ be a normal operator acting on a Hilbert space $H, E_{Q}(\cdot)$ be the spectral measure associated with $Q$ and $x \in H$. We denote by $\operatorname{supp}_{Q}(x)$ the support of the measure $\left(x \mid d E_{Q}(\cdot) x\right)$. We say that $x$ is of compact $Q$-support if $\operatorname{supp}_{Q}(x)$ is bounded (hence compact). Clearly the concept of compact $Q$ support and that of compact $|Q|$-support coincide.

The link between (5) and Definition 2.1 is given by the following.

Theorem 2.4. Let $R$ and $S$ be normal operators acting on a Hilbert space $H$. Then the following conditions are equivalent:

1. $(R, S)$ is a $(p, q)$-commuting pair,

2. The set $\mathscr{D}$ of all vectors with compact $R$ and $S$ support is dense, invariant under the action of $R, R^{*}, S, S^{*}$ and

$$
\begin{gathered}
R S x=p S R x, \\
R S^{*} x=q S^{*} R x,
\end{gathered}
$$

for any $x \in \mathscr{D}$.

3. There exists a dense domain $\mathscr{D}$ consisting of vectors with compact $R$ and $S$-support invariant under the action of $R, R^{*}, S$ and $S^{*}$ such that relations (17) hold for any $x \in D$.

Proof.

$1 \Rightarrow 2$ : The density of $\mathscr{D}$ follows from the strong commutativity of $|R|$ and $|S|$. To show that $\mathscr{D}$ is $R, S, R^{*}$ and $S^{*}$-invariant it is sufficient to notice that due to the Conditions 3 and 4 of Definition 2.1:

$\operatorname{supp}_{|R|}(($ Phase $S) x) \subset \sqrt{p / q} \operatorname{supp}_{|R|}(x), \quad \operatorname{supp}_{|R|}\left((\text { Phase } S)^{* *} x\right) \subset \sqrt{q / p} \operatorname{supp}_{|R|}(x)$ $\operatorname{supp}_{|S|}((\operatorname{Phase} R) x) \subset \sqrt{1 / p q} \operatorname{supp}_{|S|}(x), \quad \operatorname{supp}_{|S|}\left((\operatorname{Phase} R)^{*} x\right) \subset \sqrt{p q} \operatorname{supp}_{|S|}(x)$.

The trivial verification of (17) is left to the reader.

$2 \Rightarrow 3$ : trivial.

$3 \Rightarrow 1$ : It follows easily from (17) that

$$
\begin{gathered}
R^{*} S x=q^{-1} S R^{*} x, \\
R^{*} S^{*} x=p^{-1} S^{*} R^{*} x,
\end{gathered}
$$


for any $x \in \mathscr{D}$. Using (17) and (18) one can easily verify that for any nonnegative integers $k, l, r$ and $s$ we have

$$
\left(R^{*}\right)^{r} R^{k+r}\left(S^{*}\right)^{s} S^{l+s} x=\left(a^{k} S^{*}\right)^{s}\left(a^{k} S\right)^{l+s}\left(b^{l} R^{*}\right)^{r}\left(b^{l} R\right)^{k+r} x,
$$

where $a=\sqrt{p q}$ and $b=\sqrt{p / q}$. It follows that

$(\text { Phase } R)^{k} f(|R|)(\text { Phase } S)^{l} g(|S|) x=(\text { Phase } S)^{l} g\left(a^{k}|S|\right)(\text { Phase } R)^{k} f\left(b^{l}|R|\right) x$,

where $f(\lambda)$ is a linear combinations of monomials $\lambda^{k+2 r}(r=0,1,2, \ldots)$ and $g(\lambda)$ is a linear combinations of monomials $\lambda^{l+2 s}(s=0,1,2, \ldots)$. Remembering that $x$ is of compact $|R|$ - and $|S|$-support and that the set of polynomials is dense in $L^{2}(v)$ for any measure $v$ with compact support we see that

$(\text { Phase } R)^{k} f(|R|)(\text { Phase } S)^{l} g(|S|)=(\text { Phase } S)^{l} g\left(a^{k}|S|\right)(\text { Phase } R)^{k} f\left(b^{l}|R|\right)$,

holds for any bounded measurable functions $f$ and $g$ on $\mathbf{R}^{+}$such that $f(0)=$ $g(0)=0$. Considering the following particular cases

1. $k=l=0$;

2. $k=l=1, \quad f(\lambda)=g(\lambda)=1$ for $\lambda>0$;

3. $k=1, l=0, f(\lambda)=1$ for $\lambda>0$;

4. $k=0, l=1, g(\lambda)=1$ for $\lambda>0$;

we see that Conditions $1,2,3$ and 4 of Definition 2.1 are satisfied. Q.E.D.

\section{§3. The Hilbert Space Level}

Motivated by Theorem 2.4 we introduce

Definition 3.1. Let $\tilde{\alpha}, \tilde{\beta}, \tilde{\gamma}$ and $\tilde{\delta}$ be closed operators acting on a Hilbert space $\tilde{H}$. We say that $(\tilde{\alpha}, \tilde{\beta}, \tilde{\gamma}, \tilde{\delta})$ satisfies the relations (2) and (3) if

1. $\tilde{\alpha}, \tilde{\beta}, \tilde{\gamma}$ and $\tilde{\delta}$ are normal,

2. $(\tilde{\alpha}, \tilde{\beta})$ and $(\tilde{\gamma}, \tilde{\delta})$ are $(\mu, \mu)$-commuting pairs,

3. $(\tilde{\alpha}, \tilde{\gamma})$ and $(\tilde{\beta}, \tilde{\delta})$ are $\left(\mu, \mu^{-1}\right)$-commuting pairs,

4. $(\tilde{\beta}, \tilde{\gamma})$ is a $(1,1)$-commuting pair,

5. For any $x \in \mathscr{D}(\tilde{\delta}) \cap \mathscr{D}(\tilde{\beta} \tilde{\gamma})$ we have $\tilde{\delta} x \in \mathscr{D}(\tilde{\alpha})$ and

$$
\tilde{\alpha} \tilde{\delta} x-\mu \tilde{\beta} \tilde{\gamma} x=x .
$$

6. For any $x \in \mathscr{D}(\tilde{\alpha}) \cap \mathscr{D}(\tilde{\beta} \tilde{\gamma})$ we have $\tilde{\alpha} x \in \mathscr{D}(\tilde{\delta})$ and

$$
\tilde{\delta} \tilde{\alpha} x-\mu^{-1} \tilde{\beta} \tilde{\gamma} x=x .
$$

We have

Theorem 3.2. Let $(\tilde{\alpha}, \tilde{\beta}, \tilde{\gamma}, \tilde{\delta})$ be a quadruple of closed operators satisfying the relations (2) and (3). Then 
1. $\operatorname{Sp}(\tilde{\beta} \tilde{\gamma}) \subset \overline{\mathbb{C}}^{\mu}$

2. $\operatorname{ker} \tilde{\delta}=\{0\}$ and $\tilde{\delta}^{-1}=F_{\mu}(\mu \tilde{\beta} \tilde{\gamma}) \tilde{\alpha} F_{\mu}(\mu \tilde{\beta} \tilde{\gamma})^{*}$

3. $\tilde{\alpha}=\tilde{\delta}^{-1} \dot{+} \mu \tilde{\beta} \tilde{\gamma} \tilde{\delta}^{-1}$.

Proof. Let $y \in \operatorname{ker} \tilde{\delta} \cap \mathscr{D}(\tilde{\beta} \tilde{\gamma})$, then $\tilde{\delta}^{*} y=\tilde{\delta} y=0$. By virtue of Condition 5 of Definition 3.1: $\tilde{\beta} \tilde{\gamma} y=-\mu^{-1} y$. Consequently $(\tilde{\beta} \tilde{\gamma})^{*} y=-\mu^{-1} y$ and for any $x \in \mathscr{D}(\tilde{\alpha}) \cap \mathscr{D}(\tilde{\beta} \tilde{\gamma})$ we get (cf. Condition 6 of Definition 3.1)

$$
\begin{aligned}
(y \mid x) & =\left(y \mid \tilde{\delta} \tilde{\alpha} x-\mu^{-1} \tilde{\beta} \tilde{\gamma} x\right) \\
& =\left(\tilde{\delta}^{*} y \mid \tilde{\alpha} x\right)-\mu^{-1}\left((\tilde{\beta} \tilde{\gamma})^{*} y \mid x\right)=\mu^{-2}(y \mid x) .
\end{aligned}
$$

Therefore $(y \mid x)=0$ and remembering that $\mathscr{D}(\tilde{\alpha}) \cap \mathscr{D}(\tilde{\beta} \tilde{\gamma})$ is dense (cf. Theorem 2.4.3; $(\tilde{\alpha}, \tilde{\beta} \tilde{\gamma})$ is a $\left(\mu^{2}, 1\right)$-commuting pair) we see that $\operatorname{ker} \tilde{\delta} \cap \mathscr{D}(\tilde{\beta} \tilde{\gamma})=\{0\} . \quad$ On the other hand we know (cf. Conditions 2, 3 and 4 of Definition 3.1) that $|\tilde{\delta}|$ and $|\tilde{\beta} \tilde{\gamma}|$ strongly commute. Therefore $\operatorname{ker} \tilde{\delta} \cap \mathscr{D}(\tilde{\beta} \tilde{\gamma})$ is dense in $\operatorname{ker} \tilde{\delta}$ and $\operatorname{ker} \tilde{\delta}=\{0\}$.

It means that $\tilde{\delta}$ is invertible: $\tilde{\delta}^{-1}$ is an unbounded normal operator. By virtue of $(13),(\tilde{\beta} \tilde{\gamma}, \tilde{\delta}) \in \mathscr{C}\left(\mu^{2}, 1\right)$. Using (11) and (10) we get $\left(\tilde{\delta}^{-1}, \tilde{\beta} \tilde{\gamma}\right) \in \mathscr{C}\left(\mu^{2}, 1\right)$.

Let $y \in \mathscr{D}\left(\tilde{\delta}^{-1}\right) \cap \mathscr{D}\left(\tilde{\delta}^{-1} \tilde{\beta} \tilde{\gamma}\right)$. Then $y=\tilde{\delta} x$ where $x \in \mathscr{D}(\tilde{\delta}) \cap \mathscr{D}(\tilde{\beta} \tilde{\gamma})$ and using Condition 5 of Definition 3.1 we see that $y \in \mathscr{D}(\tilde{\alpha})$ and $\tilde{\alpha} y=x+\mu \tilde{\beta} \tilde{\gamma} \mathbf{x}=\tilde{\delta}^{-1} y+$ $\mu \tilde{\beta} \tilde{\gamma} \tilde{\delta}^{-1} y$. It shows that

$$
\tilde{\alpha} \supset \tilde{\delta}^{-1}+\mu \tilde{\beta} \tilde{\gamma} \tilde{\delta}^{-1}
$$

Using Theorem 2.3 with $R$ and $S$ replaced by $\mu \tilde{\beta} \tilde{\gamma} \tilde{\delta}^{-1}$ and $\tilde{\delta}^{-1}$ and remembering that normal operators have no proper normal extensions we get

$$
\begin{gathered}
\tilde{\alpha}=\tilde{\delta}^{-1} \dot{+} \mu \tilde{\beta} \tilde{\gamma} \tilde{\delta}^{-1}, \\
\operatorname{Sp} \mu \tilde{\beta} \tilde{\gamma} \subset \overline{\mathbb{C}}^{\mu}, \\
\tilde{\alpha}=F_{\mu}(\mu \tilde{\beta} \tilde{\gamma})^{*} \tilde{\delta}^{-1} F_{\mu}(\mu \tilde{\beta} \tilde{\gamma}) .
\end{gathered}
$$

Now statements 1,2 , and 3 follow immediately.

Q.E.D.

The reader certainly noticed that Definition 3.1 takes into account only 16 out of 17 relations (2) and (3): the relation " $\alpha \delta^{*}=\delta^{*} \alpha$ " seems to be forgotten. It turns out however that it may be derived from other relations if we know that $\delta$ is invertible: Assuming the nine relations

$$
\begin{aligned}
& \beta \delta=\mu \delta \beta, \quad \gamma \delta=\mu \delta \gamma, \quad \beta \gamma=\gamma \beta, \\
& \beta \delta^{*}=\mu^{-1} \delta^{*} \beta, \gamma \delta^{*}=\mu \delta^{*} \gamma, \quad \beta \gamma^{*}=\gamma^{*} \beta, \\
& \beta \beta^{*}=\beta^{*} \beta, \quad \gamma \gamma^{*}=\gamma^{*} \gamma, \quad \delta \delta^{*}=\delta^{*} \delta
\end{aligned}
$$

and setting $\alpha=\delta^{-1}+\mu \beta \gamma \delta^{-1}$ one may easily derive the remaining seven relations (2) and (3). 


\section{§4. Gauss Decomposition}

According to (4)

$$
\Phi(\delta)=\gamma \otimes \beta+\delta \otimes \delta .
$$

Therefore we may expect that $\gamma \otimes \beta+\delta \otimes \delta$ is a normal element or at least has a normal extension. However (cf. Theoem 2.2) this is the case only if $\operatorname{Sp}\left(\gamma \delta^{-1} \otimes \beta \delta^{-1}\right) \subset \overline{\mathbb{C}}^{\mu}$. One can easily check that (except the trivial case, when either $\beta$ or $\gamma$ vanishes) the latter is equivalent to

$$
\begin{aligned}
& \mathrm{Sp}\left|\gamma \delta^{-1}\right| \subset r \mu^{\mathbf{Z}} \cup\{0\}, \\
& \mathrm{Sp}\left|\beta \delta^{-1}\right| \subset r^{-1} \mu^{\mathbf{Z}} \cup\{0\},
\end{aligned}
$$

where $r$ is a positive real number. Multiplying $\beta$ and dividing $\gamma$ by $r$ (this operation preserves all the relations considered so far) we may assume that $r=1$.

The above considerations show that the satisfactory theory of the Lorentz group investigated in this paper cannot be based on the algebraic relations (2) and (3) only. We are forced to add conditions of the analytic nature. We met this phenomenon for the first time investigating quantum $E$ (2)-group (cf. [6], page 424).

Definition 4.1. Let $(\tilde{\alpha}, \tilde{\beta}, \tilde{\gamma}, \tilde{\delta})$ be a quadruple of closed operators satisfying the relations (2) and (3). We say that $(\tilde{\alpha}, \tilde{\beta}, \tilde{\gamma}, \tilde{\delta})$ satisfies the spectral condition if

$$
\begin{aligned}
& \operatorname{Sp}\left|\tilde{\gamma} \tilde{\delta}^{-1}\right| \subset \mu^{\mathbf{Z}} \cup\{0\}, \\
& \operatorname{Sp}\left|\tilde{\beta} \tilde{\delta}^{-1}\right| \subset \mu^{\mathbf{Z}} \cup\{0\} .
\end{aligned}
$$
we have

Let $\overline{\Sigma_{\mu}}$ be the closure (in $\mathbf{R}^{2}$ ) of the set $\Sigma_{\mu}=\left\{\left(s, \mu^{r}\right): s, r-\frac{s}{2} \in \mathbf{Z}\right\}$. Then
have

Theorem 4.2. Let $(\tilde{\alpha}, \tilde{\beta}, \tilde{\gamma}, \tilde{\delta})$ be a quadruple of closed operators on a Hilbert space $\tilde{H}$ satisfying the relations (2) and (3) and the spectral condition (19). Then

$$
\left.\begin{array}{l}
\tilde{\alpha}=\tilde{v} \mu^{\tilde{N} / 2} \dot{+} \tilde{n} \tilde{b} \\
\tilde{\beta}=\tilde{n} \mu^{-\tilde{N} / 2} \\
\tilde{\gamma}=\tilde{v}^{*} \tilde{b} \\
\tilde{\delta}=\tilde{v}^{*} \mu^{-\tilde{N} / 2}
\end{array}\right\}
$$

where $\tilde{v}, \tilde{n}, \tilde{N}$ and $\tilde{b}$ are closed operators such that
(i) $\tilde{v}$ is unitary
$\left.\begin{array}{l}\text { (ii) } \tilde{n} \text { is normal and } \operatorname{Sp} \tilde{n} \subset \overline{\mathbf{C}}^{\mu} \\ \text { (iii) } \tilde{v} \tilde{n} \tilde{v}^{*}=\mu \tilde{n}\end{array}\right\}$ 
(i) $\tilde{N}$ is selfadjoint

(ii) $\tilde{b}$ is normal

(iii) $\tilde{N}$ and $|\tilde{b}|$ strongly commute

(iv) The joint spectrum

$$
\operatorname{Sp}(\tilde{N},|\tilde{b}|) \subset \overline{\Sigma_{\mu}}
$$

(v) On $(\operatorname{ker} \tilde{b})^{\perp}$

$$
(\text { Phase } \tilde{b})^{*} \tilde{N}(\text { Phase } \tilde{b})=\tilde{N}+2 I
$$

Any element of the pair $(\tilde{v}, \tilde{n})$ strongly commutes with any element of the pair $(\tilde{N}, \tilde{b})$.

The elements $\tilde{v}, \tilde{n}, \tilde{N}$ and $\tilde{b}$ are uniquely determined by $\tilde{\alpha}, \tilde{\beta}, \tilde{\gamma}$ and $\tilde{\delta}$.

Conversely if $\tilde{v}, \tilde{n}, \tilde{N}$ and $\tilde{b}$ are closed operators satisfying relations (21), (22) and (23) then the quadruple $(\tilde{\alpha}, \tilde{\beta}, \tilde{\gamma}, \tilde{\delta})$ of operators introduced by (20) satisfies the relations (2) and (3) and the spectral condition (19).

Moreover for any non-degenerate $C^{*}$-algebra $\tilde{A}$ acting on $\tilde{H}$

$$
(\tilde{\alpha}, \tilde{\beta}, \tilde{\gamma}, \tilde{\delta} \eta \tilde{A}) \Leftrightarrow(\tilde{v}, \tilde{n}, \tilde{N}, \tilde{b} \eta \tilde{A}) .
$$

Remark. The best way to memorize the relations (20) is to write them in the matrix form:

$$
\left(\begin{array}{cc}
\tilde{\alpha}, & \tilde{\beta} \\
\tilde{\gamma}, & \tilde{\delta}
\end{array}\right)=\left(\begin{array}{cc}
\tilde{v}, & \tilde{n} \\
0, & \tilde{v}^{*}
\end{array}\right)\left(\begin{array}{cc}
\mu^{\tilde{N} / 2}, & 0 \\
\tilde{b}, & \mu^{-\tilde{N} / 2}
\end{array}\right) .
$$

The first matrix on the right hand side is of the form of basic representation of $E_{\mu}(2)$, whereas the second one is in the same way related to $\hat{E}_{\mu}(2)$ (cf. [6] and [8]). We call (24) the Gauss decomposition (cf. [2]).

Proof. We know (cf. Theorem 3.2.2) that $\tilde{\delta}$ is invertible. Comparing (19) with Theorem 3.2.1 one can easily show that $\operatorname{Sp} \tilde{\delta}^{2} \subset \overline{\mathbb{C}}^{\mu}$. Therefore the polar decomposition of $\tilde{\delta}$ may be written in the following form

$$
\tilde{\delta}=\tilde{v}^{*} \mu^{-\tilde{N} / 2}
$$

where $\tilde{v}$ is a unitary and $\tilde{N}$ is a selfadjoint operator with integer spectrum and $\tilde{v}$ strongly commutes with $\tilde{N}$.

We know that $(\tilde{\gamma}, \tilde{\delta}) \in \mathscr{C}(\mu, \mu)$. Therefore (cf. Conditions 2 and 4 of Definition 2.1) $\tilde{v}^{*}=$ Phase $\tilde{\delta}$ strongly commutes with $\tilde{\gamma}$ and

$$
\tilde{b}=\tilde{v} \tilde{\gamma}
$$

is a normal operator strongly commuting with $\tilde{v}$. Moreover $(\tilde{b},|\tilde{\delta}|)=\left(\tilde{b}, \mu^{-\tilde{N} / 2}\right) \epsilon$ $\mathscr{C}(\mu, \mu)$ and relations (iii) and (v) of (22) follow immediately. Remembering that $\operatorname{Sp} \tilde{N} \subset \mathbb{Z}$ and using the first inclusion (19) we get relation (iv) of (22). 
By virtue of $(9)$ and $(11),(\tilde{\beta},|\tilde{\delta}|) \in \mathscr{C}(1,1)$ and $\left(\tilde{\beta},|\tilde{\delta}|^{-1}\right) \in \mathscr{C}(1,1)$. Therefore

$$
\tilde{n}=\tilde{\beta}|\tilde{\delta}|^{-1}=\tilde{\beta} \mu^{\tilde{N} / 2}
$$

is a normal operator strongly commuting with $\tilde{N}$. According to the second inclusion (19) $\operatorname{Sp} \tilde{n} \subset \overline{\mathbf{C}}^{\mu}$. Combining the relation $(\tilde{\beta}, \tilde{\delta}) \in \mathscr{C}\left(\mu, \mu^{-1}\right)$ with the


$\mathscr{C}\left(\mu, \mu^{-1}\right)$ and relation (iii) of (21) follows.

Using in turn (8), (7), (12) and (10) we get $\left(\tilde{\beta}, \tilde{v}^{*}\right) \in \mathscr{C}\left(\mu, \mu^{-1}\right),(\tilde{\beta}, \tilde{v}) \in$ $\mathscr{C}\left(\mu^{-1}, \mu\right),(\tilde{\beta}, \tilde{b}) \in \mathscr{C}\left(\mu^{-1}, \mu\right)$ and $(\tilde{b}, \tilde{\beta}) \in \mathscr{C}(\mu, \mu)$. Similarly using (9), (11) and (13) we obtain $(\tilde{\gamma},|\tilde{\delta}|) \in \mathscr{C}(\mu, \mu),\left(\tilde{\gamma},|\tilde{\delta}|^{-1}\right) \in \mathscr{C}\left(\mu^{-1}, \mu^{-1}\right)$ and $\left(\tilde{b},|\tilde{\delta}|^{-1}\right) \in \mathscr{C}\left(\mu^{-1}, \mu^{-1}\right)$. Using now (12) we get $(\tilde{b}, \tilde{n}) \in \mathscr{C}(1,1)$. It shows that $\tilde{b}$ and $\tilde{n}$ strongly commute.

The last three formulae of (20) follow immediately from (25), (26) and (27). The first one follows from Theorem 3.2.3. This way the first part of the theorem is proved.

Assume now the relations (21), (22) and (23) and consider operators $\tilde{\alpha}, \tilde{\beta}$, $\tilde{\gamma}$ and $\tilde{\delta}$ introduced by (20). The only nontrivial points of the second part of the theorem are the normality of $\tilde{\alpha}$ and the commutation relations involving Phase $\tilde{\alpha}$ and $|\tilde{\alpha}|$. Let $S=\tilde{v} \mu^{\tilde{N} / 2}=\tilde{\delta}^{-1}$ and $R=\tilde{n} \tilde{b}=\mu \tilde{\beta} \tilde{\gamma} S$. Then $(R, S) \in$ $\mathscr{C}\left(\mu^{-2}, 1\right), \operatorname{Sp}\left(R S^{-1}\right) \subset \overline{\mathbb{C}}^{\mu}$ and $\tilde{\alpha}=S \dot{+} R$. By virtue of Theorem 2.3, $\tilde{\alpha}$ is normal and

$$
\tilde{\alpha}=F_{\mu}(\mu \tilde{\beta} \tilde{\gamma})^{*} \tilde{v} \mu^{\tilde{N} / 2} F_{\mu}(\mu \tilde{\beta} \tilde{\gamma}) .
$$

Using the last formula one can easily check that $(\tilde{\alpha}, \tilde{\beta}) \in \mathscr{C}(\mu, \mu)$ and $(\tilde{\alpha}, \tilde{\gamma}) \in$ $\mathscr{C}\left(\mu, \mu^{-1}\right)$. This ends the proof of the second part of the theorem.

If $\tilde{\alpha}, \tilde{\beta}, \tilde{\gamma}, \tilde{\delta} \eta \tilde{A}$ then (cf. Theorem 3.2.2) $\tilde{\delta}^{-1} \eta \tilde{A}$. Therefore $\tilde{v}=(\text { Phase } \tilde{\delta})^{*}$ and $\tilde{N}=-2 \log _{\mu}(|\tilde{\delta}|)$ are affiliated with $\tilde{A}$ and by virtue of (26) and (27): $\tilde{n}, \tilde{b} \eta \tilde{A}$.

Conversely if $\tilde{v}, \tilde{n}, \tilde{N}, \tilde{b} \eta \tilde{A}$ then according to the last three relations (20) $\tilde{\beta}, \tilde{\gamma}, \tilde{\delta} \eta \tilde{A}$. Using (28) we get $\tilde{\alpha} \eta \tilde{A}$.

Q.E.D.

\section{§5. $\mathbb{C}^{*}$-algebra Level}

In this Section we construct the universal $C^{*}$-algebra $A$ related with (2), (3) and (19). The algebra $A$ plays the role of function algebra of $G_{\mu}$. The comultiplication $\Phi \in \operatorname{Mor}(A, A \otimes A)$ endowing $G_{\mu}$ with the group structure will be introduced.

Comparing Theorem 4.2 with the content of [8] we discover that the relations (21) and (22) coincide with the relations imposed on "generators" $v$, $n$ and $N, b$ of the function algebras $A$ and $B$ of $E_{\mu}(2)$ and $\hat{E}_{\mu}(2)$ quantum groups. In what follows we write $A_{1}$ and $A_{2}$ instead of $A$ and $B$.

The algebra $A_{1}$ is described in details in Section 1 of [8]. We know that there exists comultiplication $\Phi_{1} \in \operatorname{Mor}\left(A_{1}, A_{1} \otimes A_{1}\right)$ (denoted by $\Phi$ in [8]) such that 


$$
\left.\begin{array}{l}
\Phi_{1}(v)=v \otimes v \\
\Phi_{1}(n)=v \otimes n+n \otimes v^{*} .
\end{array}\right\}
$$

The algebra $A_{2}$ is introduced in Section 3 of [8]. It is known that there exists comultiplication $\Phi_{2} \in \operatorname{Mor}\left(A_{2}, A_{2} \otimes A_{2}\right)$ (denoted by $\hat{\Phi}$ in [8]) such that

$$
\left.\begin{array}{rl}
\Phi_{2}(N) & =N \otimes I \dot{+} \otimes N \\
\Phi_{2}(b) & =b \otimes \mu^{N / 2}+\mu^{-N / 2} \otimes b .
\end{array}\right\}
$$

Let

$$
w=F_{\mu}\left(\mu^{N / 2} b \otimes v n\right)(I \otimes v)^{N \otimes I} .
$$

Then $w$ is a unitary element of $M\left(A_{2} \otimes A_{1}\right)$ and (cf. Theorems 3.2 and 4.1 of [8])

$$
\left.\begin{array}{l}
\left(\mathrm{id} \otimes \Phi_{1}\right) w=w_{12} w_{13} \\
\left(\Phi_{2} \otimes \mathrm{id}\right) w=w_{23} w_{13}
\end{array}\right\}
$$

Let $A=A_{1} \otimes A_{2}$ and (cf. (20))

$$
\left.\begin{array}{l}
\alpha=v \otimes \mu^{N / 2} \dot{+} n \otimes b \\
\beta=n \otimes \mu^{-N / 2} \\
\gamma=v^{*} \otimes b \\
\delta=v^{*} \otimes \mu^{-N / 2} .
\end{array}\right\}
$$

Then $\alpha, \beta, \gamma, \delta \eta A$. Combining the Theorems 1.1 and 3.1 of [8] with Theorem 4.2 we get

Theorem 5.1. 1. Let $\pi$ be a representation of $A$ in a Hilbert space $\tilde{H}$ and

$$
\left.\begin{array}{ll}
\tilde{\alpha}=\pi(\alpha), & \tilde{\beta}=\pi(\beta), \\
\tilde{\gamma}=\pi(\gamma), & \tilde{\delta}=\pi(\delta) .
\end{array}\right\}
$$

Then the quadruple $(\tilde{\alpha}, \tilde{\beta}, \tilde{\gamma}, \tilde{\delta})$ satisfies the relations (2), (3) and the spectral condition (19).

2. Any quadruple $(\tilde{\alpha}, \tilde{\beta}, \tilde{\gamma}, \tilde{\delta})$ of closed operators on a Hilbert space $\tilde{H}$ satisfying the relations (2), (3) and (19) is of the form (34), where $\pi$ is a representation of $A$ in $\tilde{H}$. The representation $\pi$ is uniquely determined by $(\tilde{\alpha}, \tilde{\beta}, \tilde{\gamma}, \tilde{\delta})$.

3. Let $\pi, \tilde{H}, \tilde{\alpha}, \tilde{\beta}, \tilde{\gamma}, \tilde{\delta}$ be as in Statement 1 and $\tilde{A} \in C^{*}(\tilde{H})$. Then

$$
(\tilde{\alpha}, \tilde{\beta}, \tilde{\gamma}, \tilde{\delta} \eta \tilde{A}) \Leftrightarrow(\pi \in \operatorname{Mor}(A, \tilde{A})) .
$$

This theorem shows that the algebra $A$ is the one corresponding to the commutation relations (2), (3) and (19). Copying formulae (4.9) and (4.16) of [5] we set:

$$
\Phi=(\mathrm{id} \otimes \sigma \otimes \mathrm{id})\left(\Phi_{1} \otimes \Phi_{2}\right),
$$


where $\sigma \in \operatorname{Mor}\left(A_{1} \otimes A_{2}, A_{2} \otimes A_{1}\right)$ is introduced by the formula

$$
\sigma(a \otimes x)=w(x \otimes a) w^{*}
$$

for any $a \in A_{1}$ and $x \in A_{2}$. Clearly $\Phi \in \operatorname{Mor}(A, A \otimes A)$. Repeating the computations of [5] (pp. 406-408) one can easily show that $\Phi$ is coassociative. We shall prove

Theorem 5.2. The action of $\Phi$ on the distinguished elements $\alpha, \beta, \gamma, \delta \eta A$ is described by the standard formulae:

$$
\begin{aligned}
& \Phi(\alpha)=\alpha \otimes \alpha \dot{+} \beta \otimes \gamma \\
& \Phi(\beta)=\alpha \otimes \beta \dot{+} \beta \otimes \delta \\
& \Phi(\gamma)=\gamma \otimes \alpha \dot{+} \delta \otimes \gamma \\
& \Phi(\delta)=\gamma \otimes \beta \dot{+} \delta \otimes \delta .
\end{aligned}
$$

Proof. By virtue of the second part of Theorem 4.4 of [5] it is sufficient to show that

$$
(\mathrm{id} \otimes \sigma)\left[\left(\begin{array}{cc}
v, & n \\
0, & v^{*}
\end{array}\right) \oplus\left(\begin{array}{cc}
\mu^{N / 2}, & 0 \\
b, & \mu^{-N / 2}
\end{array}\right)\right]=\left(\begin{array}{cc}
\mu^{N / 2}, & 0 \\
b, & \mu^{-N / 2}
\end{array}\right) \oplus\left(\begin{array}{cc}
v, & n \\
0, & v^{*}
\end{array}\right)
$$

or in the expanded form

$$
\begin{aligned}
\sigma\left(v \otimes \mu^{N / 2} \dot{+} n \otimes b\right) & =\mu^{N / 2} \otimes v \\
\sigma\left(n \otimes \mu^{-N / 2}\right) & =\mu^{N / 2} \otimes n \\
\sigma\left(v^{*} \otimes b\right) & =b \otimes v \\
\sigma\left(v^{*} \otimes \mu^{-N / 2}\right) & =b \otimes n \dot{+} \mu^{-N / 2} \otimes v^{*} .
\end{aligned}
$$

These relations are equivalent to

$$
\begin{aligned}
\mu^{N / 2} \otimes v \dot{+} b \otimes n & =w^{*}\left(\mu^{N / 2} \otimes v\right) w \\
w\left(\mu^{-N / 2} \otimes n\right) w^{*} & =\mu^{N / 2} \otimes n \\
w\left(b \otimes v^{*}\right) w^{*} & =b \otimes v \\
w\left(\mu^{-N / 2} \otimes v^{*}\right) w^{*} & =b \otimes n \dot{+} \mu^{-N / 2} \otimes v^{*} .
\end{aligned}
$$

To prove (36) and (37) it is sufficient to notice that $(I \otimes v)^{N \otimes I}\left(\mu^{-N / 2} \otimes n\right) \times$ $(I \otimes v)^{-N \otimes I}=\mu^{N / 2} \otimes n$ and $(I \otimes v)^{N \otimes I}\left(b \otimes v^{*}\right)(I \otimes v)^{-N \otimes I}=b \otimes v$, whereas $\mu^{N / 2} b \otimes$ vn strongly commutes with $\mu^{N / 2} \otimes n$ and $b \otimes v$.

To prove $(38)$ it is sufficient to notice that $(I \otimes v)^{N \otimes I}$ commutes with $\mu^{-N / 2} \otimes v^{*}$ and then use the formula (14) with $R$ and $S$ replaced by $\mu^{-N / 2} \otimes v^{*}$ and $b \otimes n$ resp. 
Similarly to prove (35) it is sufficient to use the formula (15) with $R$ and $S$ replaced by $\mu^{N-2 I} b \otimes v^{2} n$ and $\mu^{N / 2} \otimes v$ and then use the equality: $(I \otimes v)^{-N \otimes I}\left(\mu^{N-2 I} b \otimes v^{2} n\right)(I \otimes v)^{N \otimes I}=b \otimes n$.

\section{Acknowledgment}

One of the authors (SLW) is grateful to Professor J. Fröhlich for his kind hospitality during the authors visit to the ETH Zürich.

\section{References}

[1] Baaj, S. and Jungl, P., Théorie bivariant de Kasparow et opérateurs non bornés dans les $C^{*}$-modules hilbertiens, C. R. Acad. Sci. Paris, Série I, 296 (1983), 875-878, see also Baaj, S., Multiplicateurs non bornés, Thése $3^{\text {éme }}$ Cycle, Université Paris VI, 11 Decembre 1980.

[2] Barut, A. O. and Racczka, R., Theory of group representations and applications, Warszawa PWN-Polish Scientific Publishers 1977.

[3] Drinfel'd V. G., Quantum groups, in Proceedings of the International Congress of Mathematicians, Berkeley 1986, American Mathematical Society, (1987), 798-820.

[4] Faddeev, L., Reshetikhin, N. and Takhtajan, L., Quantum Groups, in: Braid group, knot theory and statistical mechanics, Ed. C. N. Yang, M. L. Ge, World scientific, (1989), 97110.

[5] Podleś, P. and Woronowicz, S. L., Quantum deformation of Lorentz group, Commun. Math. Phys., 130 (1990), 381-431.

[6] Woronowicz, S. L., Unbounded elements affiliated with $C^{*}$-algebras and non-compact quantum groups, Commun. Math. Phys., 136 (1991), 399-432.

[7] - Operator equalities related to quantum E(2) group, Commun. Math. Phys., Commun. Math. Phys., 144 (1992), 417-428.

[8] —, Quantum E(2)-group and its Pontryagin dual, Lett. Math. Phys., 23 (1991), 251-263.

[9] Woronowicz, S. L. and Zakrzewski, S., Quantum deformations of Lorentz group. Hopf *-algebra level, submitted to Compositio Mathematica. 